\title{
Development of Concepts of Mathematical Functions: A Quasi- experimental Study of Fourth- and Fifth-Grade Learners in Japan
}

\author{
Yoshiki Nisawa ${ }^{1 *}$ (10
}

${ }^{1}$ Department of Education, Faculty of Education, Bukkyo University, Kyoto, JAPAN
*Corresponding Author: nisawa@bukkyo-u.ac.jp

Citation: Nisawa, Y. (2021). Development of Concepts of Mathematical Functions: A Quasi-experimental Study of Fourth- and Fifth-Grade Learners in Japan. International Electronic Journal of Mathematics Education, 16(3), em0656. https://doi.org/10.29333/iejme/11197

\begin{abstract}
ARTICLE INFO ABSTRACT
Received: 25 Apr. 2021

Accepted: 5 Aug. 2021

According to the Programme for International Student Assessment, functions constitute an important part of mathatical literacy. In Japan, students start learning about bivariate relationships in the fourth grade (at the age of 9). However, reports suggest that elementary school students and lower-level secondary school (junior high) students have an inadequate grasp of the concept; therefore, an educational intervention that facilitates elementary school students' comprehension of functions is necessary. For this purpose, this paper reports a pilot study that applied an action-learning approach to two fourth graders and two fifth graders. The intervention avoided using numerical concepts so that the learners could form a qualitative understanding of bivariate relationships, which is a prerequisite for being able to solve quantitative problems. The results indicated that the intervention was effective and could facilitate the formation of bivariate qualitative relational concepts. These findings imply that the intervention could be effectively applied to elementary school classes through an actionlearning approach.
\end{abstract}

Keywords: mathematical functions, driving variables, action-learning approach, bivariate qualitative relational concepts

\section{INTRODUCTION}

Functions constitute an important part of mathematical literacy. Hence, "change and relationships" is one of the four content subscales in mathematics as defined by the Programme for International Student Assessment (PISA) of the Organisation for Economic Co-operation and Development (OECD, 2017). In Japan's educational system, functions are fully introduced to students when they begin secondary school at age 12. In this way, students learn to identify the functions and relations as well as how to express relationships in mathematical language (Ministry of Education, Culture, Sports, Science and Technology [MEXT], 2018c, p. 50). The process of learning about functions involves two core actions: (I) identifying the variables in a phenomenon and (II) observing how one variable relates to the other. As for presecondary education content, students learn about "the concept of functions" under the "change and relationships" content subscale from the fourth to sixth grade in elementary school (ages 9 to 12). The goal of "the concept of functions" is for students to gain the core competencies that are necessary to comprehend how phenomena change and to use this information to solve mathematical problems (MEXT, 2018, p. 62). Therefore, as students advance from Grade 4 to Grade 6, they are expected to develop the ability to perform actions (I) and (II).

There is a wealth of literature on functions in the context of mathematics education in Japan (Japan Society of Mathematical Education, 2010, p. 142-157). Several reports have highlighted a problem: functions are inadequately understood by elementary school students (ages 6 to 12) and lower secondary (junior high) school students (ages 12 to 15) (National Institute for Educational Policy Research, 2008, 2014, 2015, 2016, 2017, 2018; Nisawa, 2018a; Okamori, 1983; Yokochi \& Kikuchi, 1962). In particular, Yokochi and Kikuchi, (1962) alarming report suggested that students lack an understanding of the meaning of functions. The literature emphasizes the importance of gaining an understanding of functions, acquiring the ability to solve problems by learning to see phenomena quantitatively and understanding the relationship between two variables. In a study that examined the ability of lower secondary school students to perform actions (I) and (II), Nisawa (2018b) examined the performance of students from three grades: Grade 1 (12 to 13 years old, 160 students), Grade 2 (13 to 14 years old, 152 students) and Grade 3 (14 to 15 years old, 147 students). He reported that students in none of the grades sufficiently demonstrated these abilities. This finding underscores the need for intervention at the presecondary level before students start to learn functions. Specifically, it is necessary for elementary school students to actively and consciously learn actions (I) and (II) as part of developing competency in the "change and relationships" subscale. 
Regarding teaching strategies for "the concept of functions" at the elementary school level, Abramovich et al. (2019) advocated action-learning and concept motivation. In the context of mathematics teaching, "action-learning" involves students learning by working on appropriately configured real-world problems, whereas the "concept motivation" leverages students' innate curiosity to generate enthusiasm for mathematical concepts. Vidler (1977) argued that teachers should use practical examples that are relevant to students' daily lives since children's curiosity is determined by their prior experiences. Thus, the first insight from the literature regarding actions (I) and (II) is the importance of using practical examples that have relevance for everyday life.

By performing intuitive problem solving first (i.e., without using numerical values), Dixon and Moore (1996) and Moore et al. (1991) showed that intuitive understanding is necessary to generate mathematical solution strategies. The literature also suggests the use of qualitative concepts; for example, in a study of young children, Matsuda (2002) conducted cross-sectional and longitudinal studies to clarify how they develop relational concepts of time, distance, and speed. The study emphasized the strategy of dispensing with numerical values and focusing instead on qualitative concepts, noting that "time, distance, and speed have increasing or decreasing relationships, or qualitative relationships; they lack the quantitative relationships such as proportional or inversely proportional relationships" (Matuda, 2002, p. 86). In summary, for learners to understand how to solve quantitative problems, they must first understand qualitative relational concepts. This is the second insight into teaching the core actions (I) and (II) extracted from the literature.

In Japan, the government issues curriculum guidelines (MEXT, 2018a, 2018b) that provide a framework for standardized teaching content at standardized levels for all children to be taught at the same level and to receive the same educational content. The guidelines apply to elementary, lower secondary, and upper secondary schools nationwide. Additionally, the government provides annotations that elaborate on the guidelines and provide further instruction. Elementary and lower secondary school textbooks are based on the curriculum guidelines and annotations. Accordingly, mathematics textbooks generally consist of the same content, albeit with minor variations depending on the publisher.

For elementary students in Grade 4 (9 to 10 years old), the curriculum guideline annotations indicate the following learning objectives: "1. Learner can represent changes in tables, formulas, and line graphs, and can decipher the elements of changes. 2: Learner has mastered the following thinking, decision, and expressive skills; Learner can identify and examine two inter-related quantities and can graphically or formulaically represent how the quantities change and interact" (MEXT, 2018c, p. 214). One textbook (Shimizu, 2015) provides an example of how this guideline is translated into a learning task. The stimulus for this learning task involves arranging sticks into rectangular formations. The student is required to write a formula using a square to indicate the number of sticks along the long side and a triangle to indicate the number of sticks along the short side (see Figure 1). The key issue to note about the design of this task is that it assumes that students will begin their learning of functions from quantitative numerical problems (Matsuda, 2002). Similar tasks can be found in other Japanese mathematics textbooks. As has been noted earlier, the fundamentals of functions are actions (I) and (II). Research (Dixon \& Moore, 1996; Matsuda, 2002; Moore et al., 1991) suggests that learners should first form (bivariate) qualitative relational concepts before tackling quantitative tasks, as this will lead to an understanding of functions via actions (I) and (II).

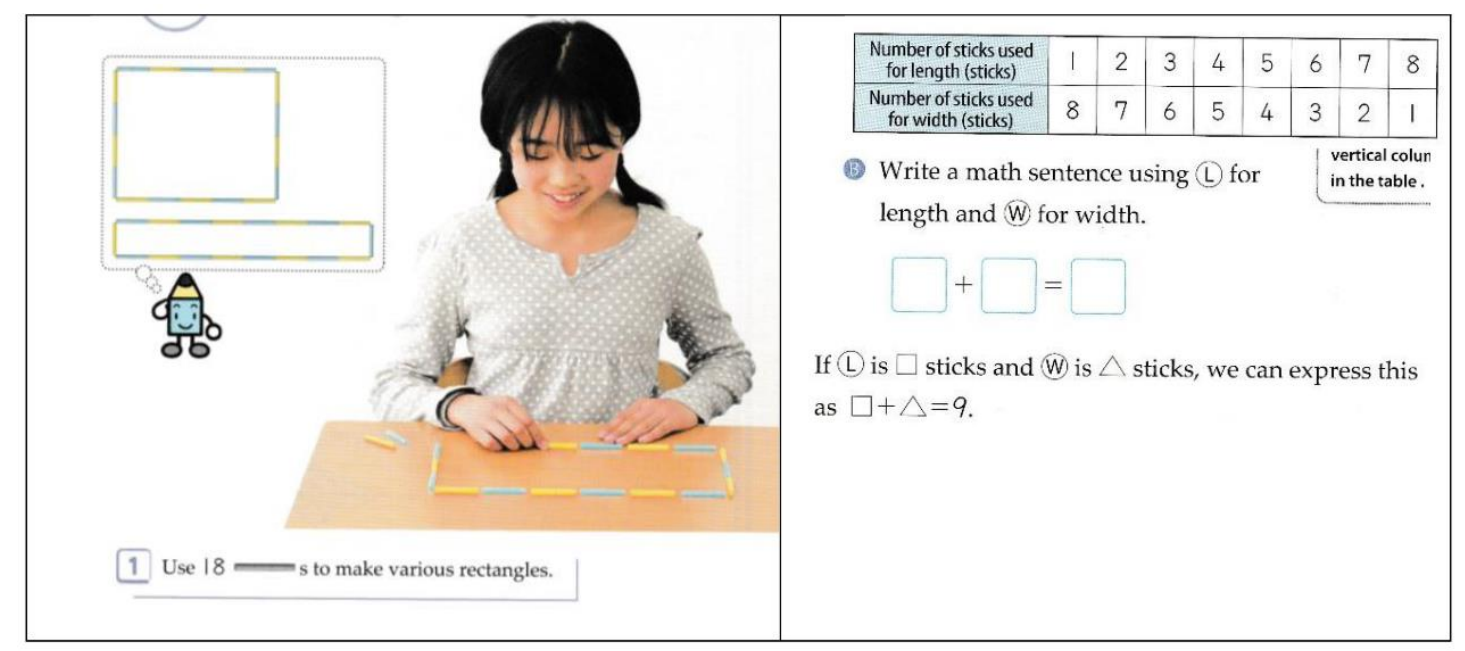

Figure 1. Example of learning content in the fourth grade of elementary school (9 to 10 years old)

For this paper, the relational concepts of time, distance and speed have been defined not only as specific variables but also as "bivariate qualitative relational concepts" (BQRCs). In a quasi-experimental study, an educational intervention design was pilottested to help elementary students learn the BQRCs underpinning actions (I) and (II). The learning task was based on the two key insights from the literature: mathematics is effectively learned using relatable, practical problems (Abramovich et al., 2019) and qualitative concepts (Dixon \& Moore, 1996; Matsuda, 2002; Moore et al., 1991). The effectiveness of the intervention was evaluated on the basis of the observations made by the students and the answers they provided in pre and postintervention tests.

\section{METHOD}

The intervention developed to help elementary school students in the fourth grade form the BQRCs necessary for performing actions (I) and (II) is described below. 
First, the intervention adopted an action-learning approach. Specifically, it involved a practical exercise in which the students observed and recorded changes in the shadow cast using a 3D object. There were various reasons for designing such a task. First, shadow changes are easy for children to relate to, as they feature in their play activities. Second, it was believed that the students could easily engage in the assignment since they had already learned about sunlight and shadows in science class in the third grade (at ages 8 to 9). Third, it was thought that the students could readily perceive the relationship between time and the shadow dimensions since the use of a conical object would create a triangular shadow, allowing for the easy measurement of sides, angles, and areas as well as the measurement of time at hourly increments. The last reason is that it was estimated that the learners could observe the relationship between two time-mediated variables (e.g., side and angle) because the conical object would cast a shadow in a triangular shape that resembles the shape of the object (see Figure 2) (Nisawa, 2018c).

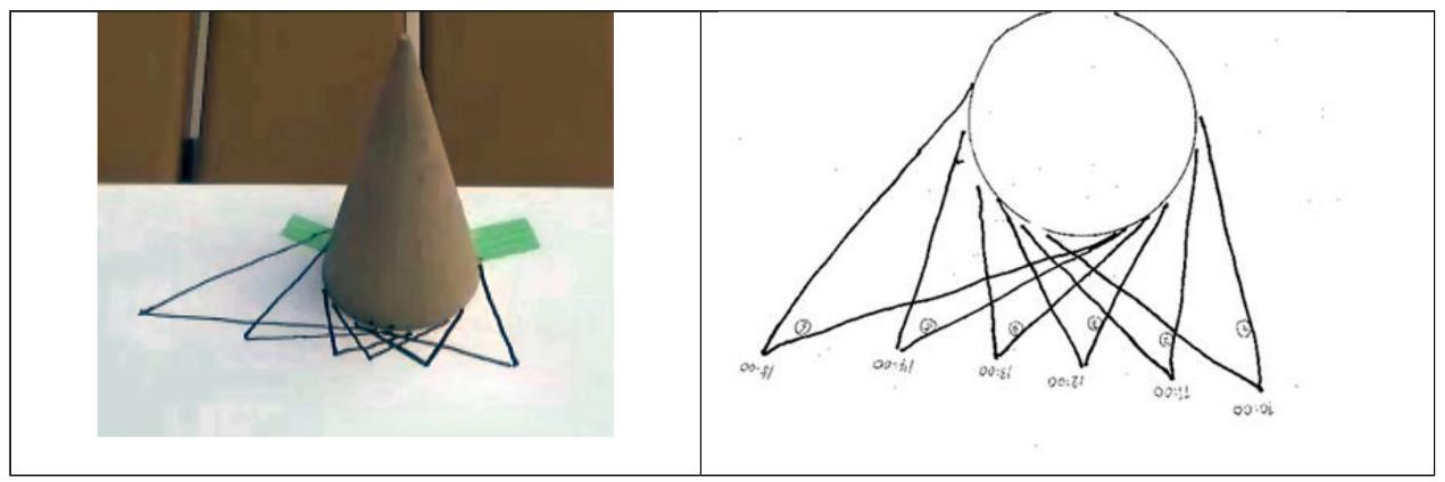

Figure 2. The recording of shadow changes (left) and the shape of the recorded shadow (right)

Next, the intervention focused on identifying variables and describing the bivariate relationships in terms of an increase or decrease, where one variable increases in value as the other decreases, or vice versa. Crucially, as per the second insight from the literature (Dixon \& Moore, 1996; Matsuda, 2002; Moore et al., 1991), the intervention avoided the use of numerical data; the intention was for learners to describe the changes in nonquantitative terms as opposed to recording numerical data of the dimension of the shadow and observing how these numerical values change.

The "change and relationships" concept into elementary school classes. Although the learning task was designed for fourth graders, the experiment also included fifth graders to determine whether the experience of learning "change and relationships" in Grade 4 affected their ability to form BQRCs for activities (I) and (II). Since this was a pilot study, it was conducted on only four students (two students from each grade) instead of on the whole class. The students involved in the pilot test were the children of university faculty members or the siblings of undergraduate students. All except one of the students disliked arithmetic. Hereafter, the two fourth graders are referred to as $A$ and $B$ and the two fifth graders as $C$ and $D$. The children were given the autonomy to decide whether to participate in the study or not, and verbally the informed consent was obtained from the children and their parents following a briefing and regarding the purpose and content of educational practice in which they were informed that no personally identifiable information would be disclosed.

Ethical approval was not required as the research in this study does not infringe or threaten the life, health, privacy, dignity and rights of the research subjects.

The procedure used during the intervention is detailed below.

While at home, each of the four students completed a preintervention test and then recorded the shadows cast by the conical object. Next, the four students gathered in a room at the university and collectively engaged in an exercise related to actions (I) and (II), during which they referred to their recorded observations. The exercise concluded with the students individually completing a postintervention test.

The purpose of the preintervention test was to evaluate whether the students perceived the passing of time in their daily lives, whether they could perceive changes quantitatively and whether they could perceive changes as BQRCs.

The questions of the preintervention test, and the logic behind them, were as follows.

Question 1: The students were presented with eight everyday situations. For each situation, they had to state whether they thought time had elapsed during that situation by selecting one of three responses: "(the time) changed," "(the time) did not change," or “I don't know the answer" (see Figure 3). 
1 Choose the appropriate expression in [ ] in the following sentences.

(1) Now, time [changed, did not change, I don't know the answer].

(2) During the Mathematics lesson, the time [changed, did not change, I don't know the answer].

(3) When you walked into the classroom, the time [changed, did not change, I don't know the answer].

(4) When we ate lunch, the time [changed, did not change, I don't know the answer].

(5) When you made a cup of tea, the time [changed, did not change, I don't know the answer].

(6) On the way to or from school, the time [changed, did not change, I don't know the answer].

(7) When you slept at night, the time [changed, did not change, I don't know the answer].

(8) In the morning on a sunny day, the time [changed, did not change, I don't know the answer].

Figure 3. Eight questions in Question 1

Question 2: The learners were presented with the image shown in Figure 4: a series of solid rectangles with dimensions that varied according to their position in the series. The students first had to identify the elements that varied across the series by answering the following question: "What things vary across the series? Write down as many things as you can". This subquestion was intended to measure their ability to perform action (I). The students then had to describe how these features changed across the series by responding to the following: "Next, describe how these things change". The purpose of the second subquestion was to measure their ability to perform action (II).

\begin{tabular}{|ccccc|}
\hline 0 & 1 Minute & 2 Minutes & 3 Minutes & 4 Minutes \\
Minute & later & later & later & later \\
& & & &
\end{tabular}

Figure 4. The figure to be considered in Question 2

Question 3: This question asked the students to perform the same two tasks from Question 2 (identify elements that vary across the series and describe how these features changed over time), but this time, the shapes in the series were replaced by the triangular silhouettes the students had previously traced. The purpose of this exercise was to gage the degree of their ability to perform actions (I) and (II) and to assess how well the students understood the spatial relationship between the sun and the conical object (see Figure 5). 


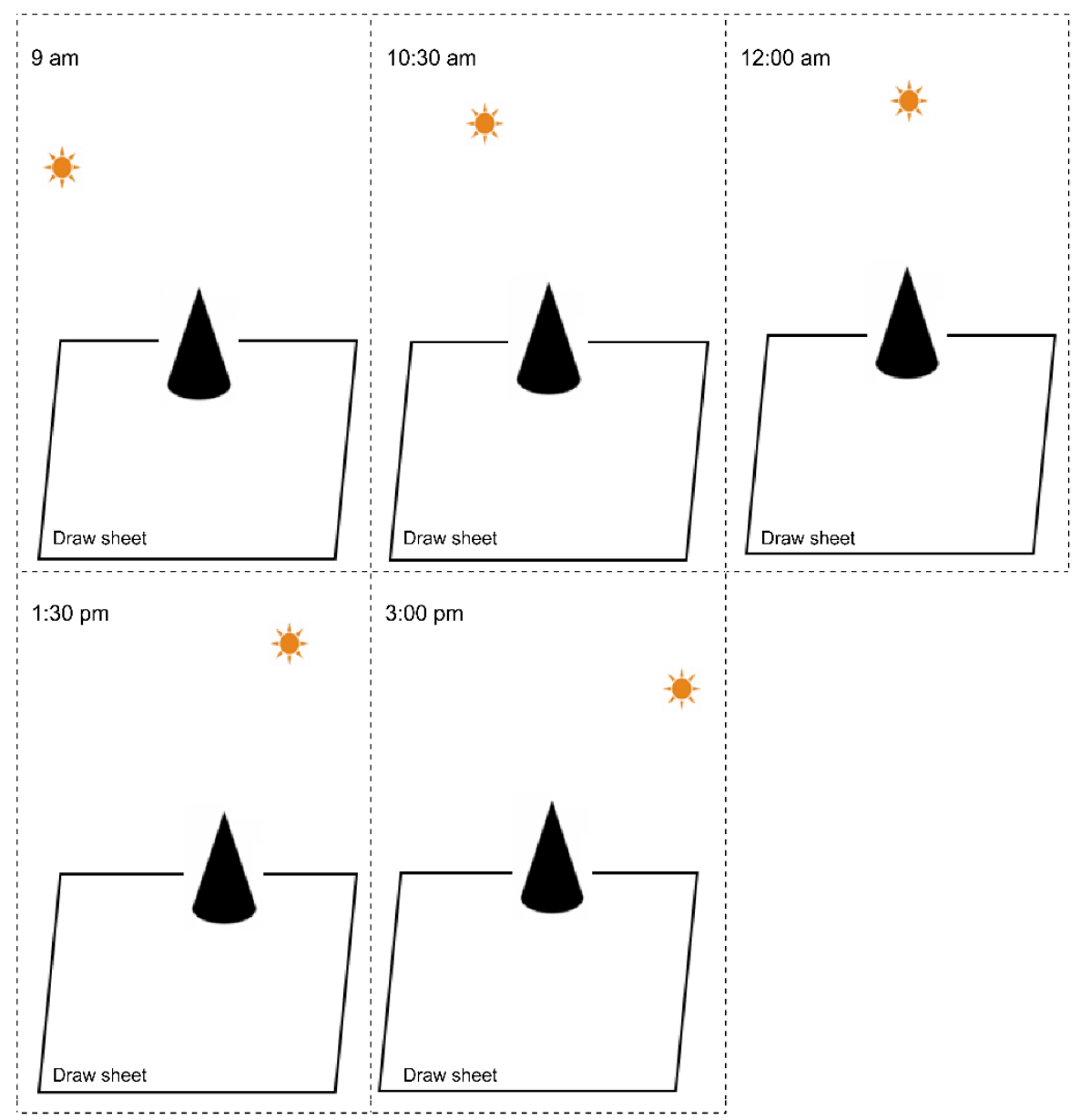

Figure 5. The position of the cone in relation to the sun in Question 3

The preintervention test instructed the students to only respond to questions that they were able to answer. The students submitted the test on the day of the class.

For the observation task, the students were given the conical object and a sheet of paper on which they would place the cone and record the shadows. The students were taught the procedure for this exercise by their parents or siblings; they put the materials outside so that the cone worked like a sundial. They traced the outline of the shadow on the paper at every hour (between 9:00 and 15:00).

The group exercise that followed the observation consisted of two lessons (Lesson 1 and Lesson 2), each of which lasted approximately 45 minutes. In Lesson 1 , the students had to identify the variables in their observations and then examine the relationship between these variables and the passing of time. In Lesson 2, they first examined the relationship between two timemediated variables and then spent around 30 minutes completing the postintervention test.

Both lessons used an active, group-work format rather than passive learning. Instead of delivering a one-way lecture, the students were observed and were encouraged through prompts and questions to share and discuss their ideas or to summarize what they had learned, allowing them to learn through student-centered dialog.

The procedures for the lessons were as follows.

\section{Lesson 1}

(i) The shape of the shadow recorded by the child on the drawing sheet is copied and cut-out (Figure 6). Variables such as length of the sides, width of the shadow, and size of the angles and area are derived. The variables are extracted on the basis of whether they can be explained by an "increase" or "decrease," and those that cannot are excluded. 


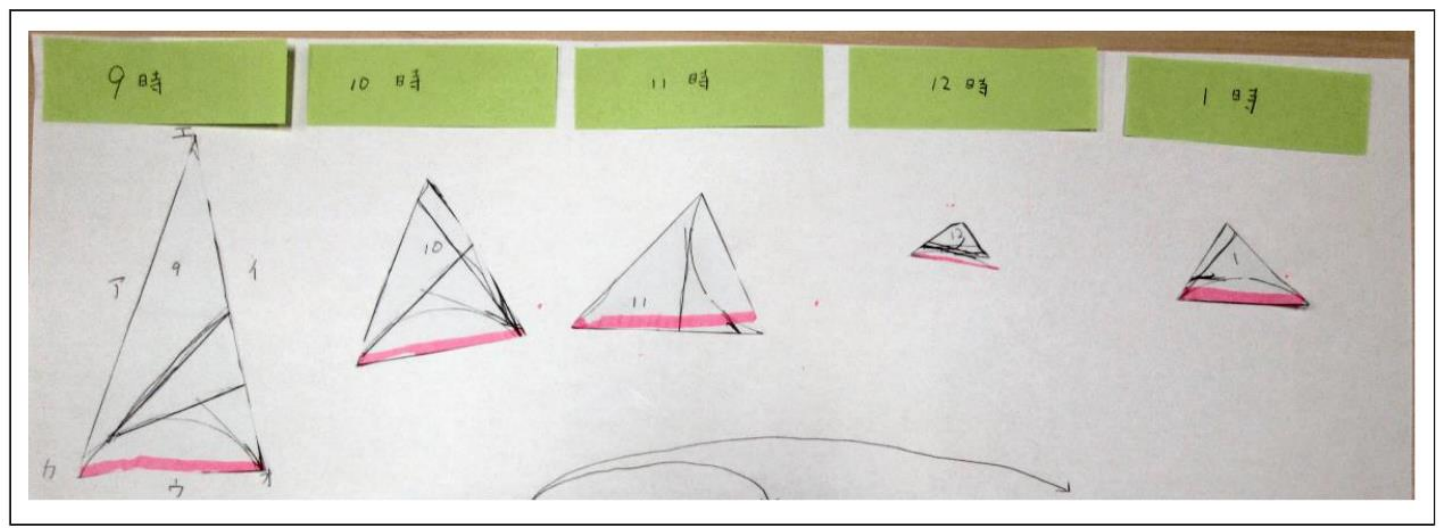

Figure 6. The shapes of the shadow recorded on the drawing sheet are arranged by time

(ii) The relationship between two variables is established. Specifically, the relationship between time and the other variables (length of sides, width of shadow, size of angles, etc.) is determined. At this stage, the child understands the correspondence between the two variables: when one variable increases, the other variable increases and when one variable increases, the other variable decreases.

\section{Lesson 2}

(iii) The relationship between two variables that involve time is established (length of sides, width of shadow, size of angles, etc.). For example, it is explained to the children that if "Side A becomes shorter as time passes," one could also say that "Side A becomes shorter because time passes"; and if "Side B becomes shorter as time passes," one could also say that "When Side A becomes shorter, side B also becomes shorter". The mathematical meaning of this is B $=g^{\circ} f^{-1}(\mathrm{~A})$ in Figure 7 .

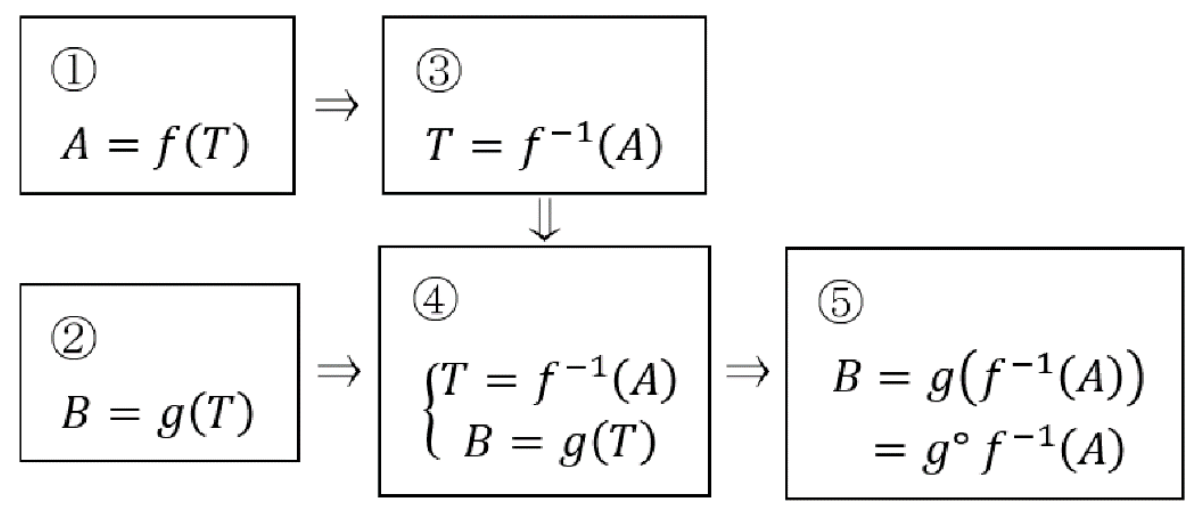

Figure 7. The mathematical meaning of (iii)

The study was held from 9:00 to 12:00 on Sunday 30 June 2019 at the university laboratory.

A postintervention test was then applied to determine whether the students had formed the BQRCs that underpin actions (I) and (II). The questions, and the thinking behind them, were as follows.

Question 1: This question was designed to prompt the learners to reflect on what they had learned in Lessons 1 and 2 and to perform actions (I) and (II) on the series of triangular silhouettes they had traced (see Figure 8). The first part of the question asked the students to indicate the elements that varied across the series ("What has changed? What is changing?"), which involved action (I). The second part asked the students to describe how these elements changed ("Let's write how it will change"), which involved action (II). 
1(i) Mirai, a fourth grader, first arranged the shapes of the shadows from 10:00 a.m. to 12:00 noon.

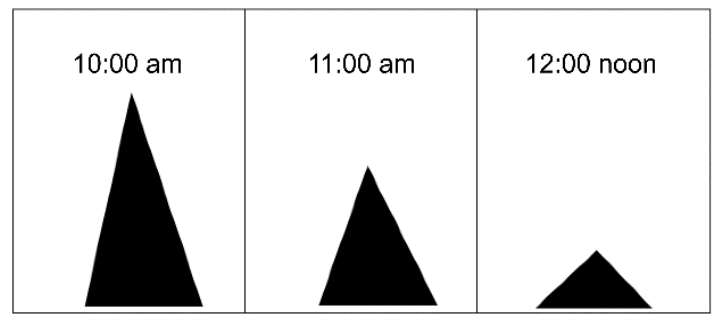

What has changed? What is changing?

Let's write how it will change.

1(ii) Mirai, a fourth grader, then arranged the shapes of the shadows from 12:00 noon to 2:00 pm.

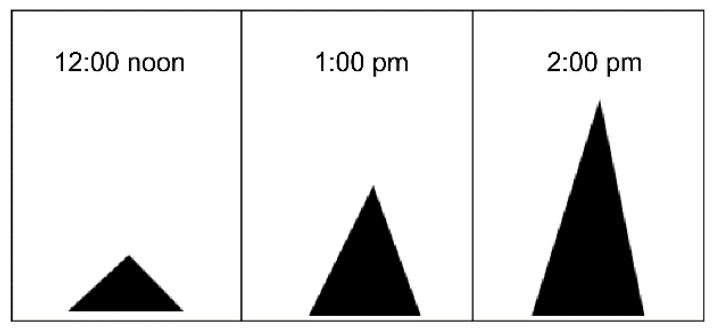

What has changed about the shapes? What is changing?

Let's write about the changes.

Figure 8. Figure showing how the shape changes in Question 1

Question 2: Like Question 1, this question tested the students' ability to perform actions (I) and (II), but this time the triangular silhouettes were replaced with rectangles (orthogonal or nonorthogonal) (see Figure 9).

2(i) The figures below show how a black figure changes.

\begin{tabular}{|l|l|}
\hline 1 Minute & 2 Minutes later \\
\hline
\end{tabular}

What has changed about the shapes? What is changing?

Let's write about the changes.

2(ii) The figures below show how a black figure changes.

\begin{tabular}{|l|l|l|}
\hline 1 Minute & 1 Minute later & 2 Minutes later \\
\hline
\end{tabular}

Let's write about the changes.

Figure 9. Figure showing how the shape changes

The effectiveness of the intervention was evaluated on the basis of the responses in the pre and postintervention tests as well as by the observations made by the students during the intervention. 


\section{RESULTS}

The responses for the preintervention test were as follows.

Question 1: All four students indicated that time had elapsed in all eight items.

Question 2: When asked what elements had changed across the series, all four students cited "time". Also cited were the length and width of the rectangle; for example, one student indicated that "(the rectangle) got wider". None of the students gave an answer that clarified the relationship between the variables.

All four students managed to trace the shadows accurately. However, in all four cases, the lengths of the shadows were drawn imprecisely.

When asked what elements changed across the series, all the students except one of the fourth graders cited time and length of the shadow. Other elements cited included the sun and the position of the shadow.

One of the fourth graders and one of the fifth graders identified the relationship between time and length, stating that the shadow gets longer or shorter (over time). Additionally, all the students, including the two who did not identify the relationship between time and length, cited some perceptual variables; for example, some indicated the (perceived) position of the sun, stating that it travels west.

The responses in the preintervention test indicated that although the students did correctly identify some of the variables, they missed some crucial points, and some of the variables they identified were of a perceptual nature (e.g., the sun's position).

Before the intervention on 30 June, the students' feedback about the observation task was sought. All four students said they enjoyed observing the shadow and were curious about the way the shadow changed. They also said that they had been looking forward to recording the position of the shadow.

The results of the exercise are as follows:

(i) Deriving the variables: First, the students cut-out triangles of the shadow shapes and arranged them. At this stage, none of the four children was able to extract the variables. Therefore, it was suggested that they stack the cut-outs on top of one another and compare them directly. By doing this, all four children were able to identify the left and right sides, the base, the width, and the angles as variables.

For example, when one child observed that, "The length of this side is getting shorter," another child pointed to another side and indicated, "This side is also getting shorter". This prompted another child to point out, "This angle is getting bigger," and so on.

The three sides and angles of the triangles were named (a), (b) and (c) to make it easier for the children to explain the variations in the variables. In particular, they were instructed to express the changes in the variables by an "increase" or "decrease". In response, the four children gradually stopped referring to changes in appearance, such as "the shape changes".

(ii) Establishing the relationship between the passing of time and other variables: It was confirmed that all four children understood that the change in the variables corresponded to the passing of time. It was also verified that they could explain the relationship between two variables, for example, "Side (b) becomes shorter as time passes". The children verbally discussed the relationship between the other variables with each other and could talk about it without any difficulty.

(iii) Establishing the relationship between two variables that involve time: It was explained to the children that "Side (a) becomes shorter as time passes" could also be expressed by saying that "Side (a) becomes shorter because time passes," and "Side (b) becomes shorter as time passes" could also be expressed by saying that "When side (a) becomes shorter, side (b) also becomes shorter". All four children immediately understood the relationship between the change in the lengths of sides (a) and (b). They were asked to write down the connection between the other variables in their notebooks and all were able to put in writing the relationships between the different sides, an angle and a side, a side and the area, and so on. Eventually, they were able to describe the relationship between the two variables that they had written about in their notebooks.

The results for the postintervention test were as follows.

Question 1: When asked to state the variables, all four students cited angle, side length, and area. The two fifth graders also cited time. One fourth-grade student and one fifth-grade student also indicated shape and size, instead of just stating the variables.

For Question 1, all four students were able to describe bivariate correlations. In their descriptions, they labeled the angles and sides with letters (e.g., Sides A, B and C) (see Figure 10). 


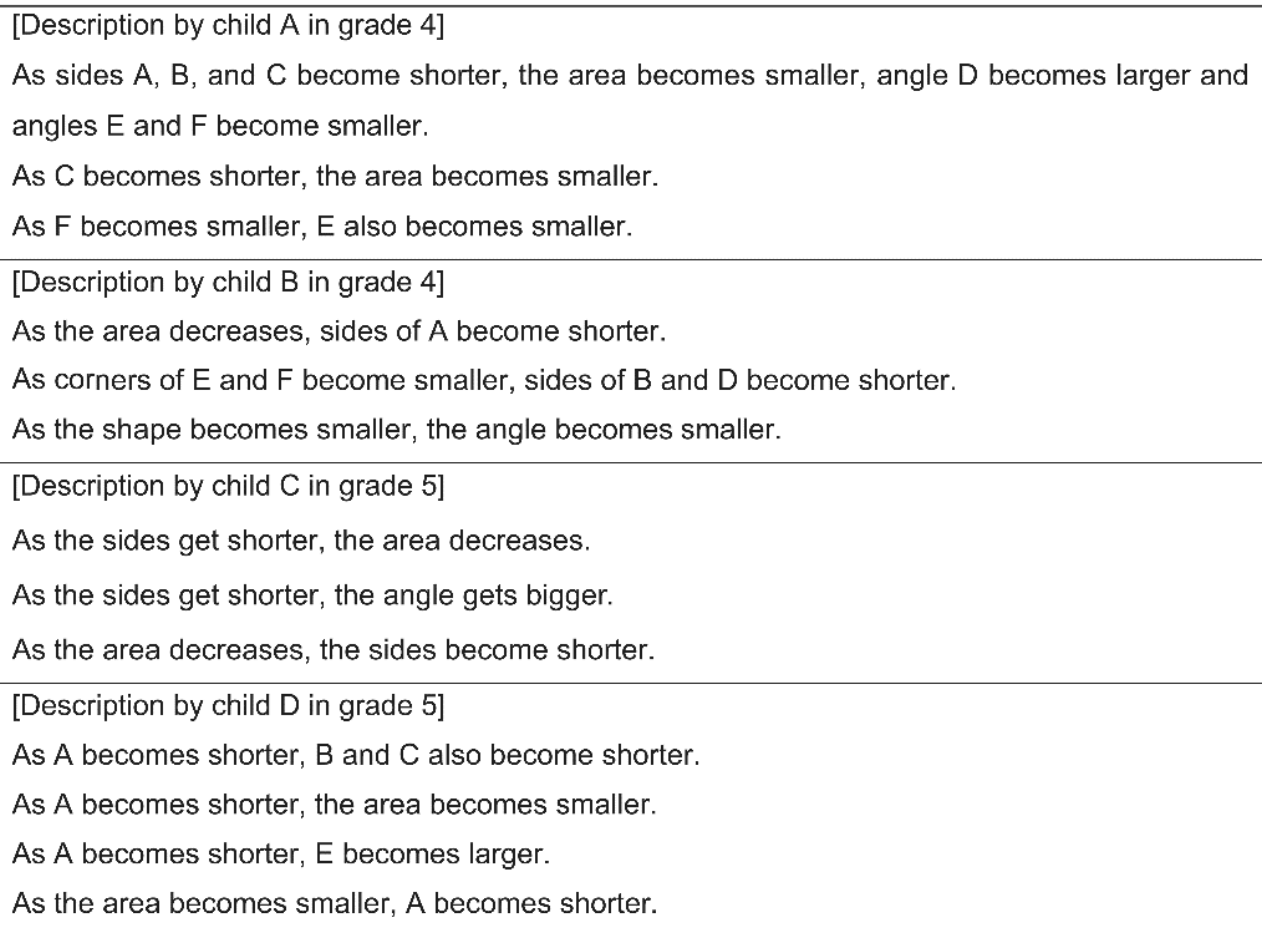

Figure 10. Children's descriptions of the situations presented in Question 1(i) (As the descriptions of the situation of Question 1 (ii) were nearly the same, they have been omitted.)

Student A described how a side's length correlates with the area, how one angle correlates with another angle and how one angle correlates with the area. Student B described how a side's length correlates with the area, how an angle correlates with the sides and how one angle correlates with another. Student $C$ described how a side's length and the area correlate with each other. Student D described how one side's length correlates with another side's length, how a side's length corresponds with an angle and how an angle corresponds with the area.

Unlike the other students, Student C (Grade 5) performed action (I) without labeling the elements alphabetically. However, the student subsequently used alphabetical labels when performing action (II). Additionally, in his/her otherwise appropriate descriptive answer, Student B (Grade 4) mentioned that the angles get smaller as the shape gets smaller, a correlation that was arguably invalid.

Question 2: For the first subquestion, all four students identified side length, angle, and surface as variables. Student D (Grade 5) additionally identified time. Student B (Grade 4) and Student D (Grade 5) described the surface as "size (of shape)" (as did Student B in Question 1) but then expressed it as a "surface" in the second subquestion.

The students described bivariate correlations using actions (I) and (II), and they labeled the sides and angles as they did in Question 1 (see Figure 11). Some of the descriptions were erroneous, such as the statement that the size of an angle increases or decreases. 


\begin{tabular}{|c|}
\hline [Description by child $\mathrm{A}$ in grade 4$]$ \\
\hline As angles $F$ and $G$ become smaller, lengths of sides $B$ and $C$ become shorter. \\
\hline As angles $\mathrm{E}$ and $\mathrm{H}$ become smaller, area becomes smaller. \\
\hline As angles $\mathrm{E}$ and $\mathrm{H}$ become smaller, angles $\mathrm{F}$ and $\mathrm{G}$ become larger. \\
\hline As angles $\mathrm{E}$ and $\mathrm{H}$ become small, sides $\mathrm{B}$ and $\mathrm{C}$ become small. \\
\hline [Description by child B in grade 4] \\
\hline As edges of sides A and B become smaller, the area also becomes smaller. \\
\hline As side $\mathrm{C}$ becomes smaller, angles $\mathrm{G}$ and $\mathrm{H}$ become smaller. \\
\hline As angles $E$ and $F$ become smaller, sides of edges $A$ and $B$ become smaller. \\
\hline [Description by child $\mathrm{C}$ in grade 5] \\
\hline As sides $B$ and $C$ become shorter, the area becomes smaller. \\
\hline As the area becomes smaller, angles $\mathrm{E}, \mathrm{F}, \mathrm{G}$, and $\mathrm{H}$ become larger. \\
\hline As the angles become larger, sides $B$ and $C$ become shorter. \\
\hline [Description by child $\mathrm{D}$ in grade 5 ] \\
\hline As sides $A, B, C$, and $D$ get shorter, the area gets smaller. \\
\hline As sides $A, B, C$, and $D$ become shorter, angles $E, F, G$, and $H$ become larger. \\
\hline As the area becomes smaller, angles $\mathrm{E}, \mathrm{F}, \mathrm{G}$, and $\mathrm{H}$ become larger. \\
\hline As the area becomes smaller, sides $\mathrm{A}, \mathrm{B}, \mathrm{C}$, and $\mathrm{D}$ become shorter. \\
\hline As angles $\mathrm{E}, \mathrm{F}, \mathrm{G}$, and $\mathrm{H}$ become large, the area becomes small. \\
\hline
\end{tabular}

Figure 11. Children's descriptions of the situations presented in Question 2(i). (The description situation of Question 2(ii) is nearly the same as that of (i), so it is omitted.)

Student A described how an angle correlates with a side's length, how an angle correlates with the surface and how one angle correlates with another. Student B described how a side's length correlates with the surface, how a side's length correlates with an angle and how the surface correlates with an angle. Student $C$ described how a side's length correlates with the surface, how the surface correlates with an angle and how an angle correlates with a side's length. Student D described how a side's length correlates with the surface, how a side's length correlates with an angle and how the surface correlates with an angle.

In the survey conducted prior to the educational exercise, the four children were unable to fully explain the driving variables and the relationship between two variables.

In the survey conducted after the educational exercise, regarding a review of the learning content, all four children were able to accurately explain the relationship between the extraction of variables and the increase and decrease of the other two variables.

By comparing the answers on the basis of the grades of the students, it appears that the fifth graders' experience of learning "change and relationships" in Grade 4 had no significant impact on their performance.

\section{DISCUSSION}

In this quasi-experimental study, an educational intervention designed to help learners from the BQRCs required for performing actions (I) and (II) was pilot-tested. The observation task-observing changes in a shadow cast by a conical objectstimulated the students' curiosity and thus generated enthusiasm for the concepts. This finding was consistent with Livson's (1967) previous suggestions about teaching mathematics to children.

The results of the preintervention test revealed that all four students recognized the passing of time in each of the everyday settings, implying that the learning content on bivariate correlations of time were adequate. Judging from their descriptive answers, the students did not fully grasp the changes in quantitative values when performing actions (I) and (II). These findings also suggest that the intervention can potentially help students form BQRCs. Additionally, there were no apparent learning differences between students from different grades, as they all gave similar answers.

The following observations were made during the intervention. The students struggled to identify the variable quantities between the cut-out shapes by viewing them in a laid-out arrangement, suggesting that other students of the same age would also struggle to do so when the items are arranged in this way. A more direct method of comparison of the cut-out shapes (MEXT, 2018, p. 56-60) would be a more effective approach to learning about measuring quantities. These observations also suggest that focusing on what increases or decreases can help the learner eliminate elements that are not variables.

Regarding the students' attempts to identify bivariate correlations, as noted in the discussion of the preintervention test results, the students had no difficulty with bivariate correlations involving time. This may be because they had gained a deep understanding of the passing of time as a result of the observation task, in which they independently recorded the shadow at 1hour intervals. Furthermore, the students already understood bivariate correlations in terms of one variable increasing as the 
other increases, or one variable decreasing as the other increases. Thus, the students demonstrated sufficient understanding of the relationship between time and other variables.

Neither did the students have any problems identifying the relationship between two time-mediated variables; each student was able to verbally express these relationships. This suggests that the students had mastered the basics of bivariate correlations (Matsuda, 2002) and were ready to actively examine correlations during the lesson. As such, the intervention method used in the pilot study was an effective way of learning about bivariate correlations.

In terms of the posttest results, all four students managed to identify variables in the stimulus and describe bivariate correlations, despite some minor differences. In their written descriptions of the bivariate correlations, the students sometimes failed to explain the correlations adequately. In their feedback following the postintervention test, they reported struggling with writing their responses, suggesting a gap between their written communication in the tests and their verbal communication during the lesson. Indeed, during the lessons, all four students managed to verbally express the correct number of correlations. Some errors were noted among the written responses to Question 2, but in each case, the student realized and understood the error after it had been pointed out to them following the test.

Thus, the students' in-lesson verbal descriptions of the bivariate correlations as well as their postintervention written responses suggest that they made progress during the educational intervention in forming BQRCs, especially given that thinking skills have a mutually complementary relationship with expressive skills (MEXT, 2008, p. 20). The results also imply that the effect of the intervention was stable across different grades. Therefore, the intervention proved to effectively facilitate the formation of BCQRs in elementary school students.

Although the intervention was only implemented with four students, it is feasible to apply this method in regular elementary school classes and to expect similar results, given that this study was primarily designed for a group-learning format.

\section{CONCLUSION}

Although students in Japan start learning mathematical functions when they reach lower secondary school, they are introduced to "the concept of functions" in the fourth grade (9 to 10 years old) as part of the "change and relationships" content subscale. As discussed, learning about functions requires two core actions: (I) identifying the variables in a phenomenon and (II) observing how one variable relates to the other. Accordingly, the intervention was designed to help learners form BQRCs for these two actions (I) and (II). Theoretical insights from the literature were also applied, including the theory that numerical concepts are more effectively mastered by working on appropriately configured real-world problems (Abramovich et al., 2019), the theory that intuitive problem solving (without the use of numerical values) and intuitive understanding are necessary to generate strategies to solve mathematical problems (Dixon \& Moore, 1996; Moore et al., 1991) and the theory that forming qualitative (nonnumerical) concepts is effective for developing numerical concepts (Matsuda, 2002). The intervention used a sample of four students-two fourth graders and two fifth graders.

The results of the study were as follows. First, the observation task (recording changes in the shadow cast by a conical object) stimulated the students' motivation. Second, the students found it easier to identify variables once they had tried to make a direct comparison, in which they overlaid the cut-out silhouettes to determine which elements had increased or decreased. Third, once they had identified the variables, the students managed to easily grasp the correlation with time and could understand that one variable increases as the other increases or that one variable increases as the other decreases. They then easily managed to describe time-mediated bivariate correlations. Fourth, the comparison of the pre and postintervention test responses revealed that students had managed to describe time-mediated bivariate correlations. Finally, the students' in-lesson verbal descriptions and their postintervention written responses in the pre and postintervention tests suggest that there was no difference between the fourth and fifth graders in their performance relative to actions (I) and (II).

The findings first suggest that the intervention method used in this study (i.e., the learning materials and the lesson) is an effective way to help learners form BQRCs. The pilot study outcome also implies that the intervention would be effective in elementary school classes using a group-work format, highlighting the value of action-learning as a teaching strategy for this content.

However, further research is necessary to demonstrate that the outcome of the intervention-the formation of BQRCsfacilitates the ability to solve numerical problems. It is also necessary to determine whether the results of this study are generalizable (i.e., whether applying the intervention in regular elementary classes with a group-work format would facilitate the formation of BQRCs). Obtaining such findings would contribute toward a framework of action for teaching mathematical functions to elementary students.

Author contributions: All authors have sufficiently contributed to the study, and agreed with the results and conclusions.

Funding: This work was supported by JSPS KAKENHI Grant Numbers 17K04823, 20 K02895.

Declaration of interest: No conflict of interest is declared by authors.

\section{REFERENCES}

Abramovich S., Grinshpan A. Z., \& Milligan D. L. (2019). Teaching mathematics through concept motivation and action learning. Education Research International, 2019. https://doi.org/10.1155/2019/3745406 
Dixon, J. A., \& Moore, C., F. (1996). The developmental role of intuitive principles in choosing mathematical strategies. Developmental Psychology, 32(2), 241-253. https://doi.org/10.1037/0012-1649.32.2.241

Japan Society of Mathematical Education (Ed.) (2010). Handbook of research in mathematics education. Toyokan Syuppan.

Livson N. (1967). Towards a differentiated construct of curiosity. The Journal of Genetic Psychology, 111(1), 73-84. https://doi.org/10.1080/00221325.1967.10533749

Matsuda, F. (2002). The development of relationship concept. Kitaoji-syobo.

Ministry of Education, Culture, Sports, Science and Technology. (2018a). Elementary school curriculum guidelines. https://www.mext.go.jp/content/1413522_001.pdf

Ministry of Education, Culture, Sports, Science and Technology. (2018b). Junior high school curriculum guidelines. https://www.mext.go.jp/content/1413522_002.pdf

Ministry of Education, Culture, Sports, Science and Technology. (2018c). Elementary school curriculum guidelines commentary mathematics. Education Publishing.

Moore C. F., Dixon J. A., \& Haines B. A. (1991). Components of understanding in proportional reasoning: a fuzzy set representation of developmental progressions. Child Development, 62(3), 441-459. https://doi.org/10.2307/1131122

National Institute for Educational Policy Research. (2008). Curriculum Research Center "National Assessment of Academic Ability" Assessment questions, correct answer examples, explanatory materials. https://www.nier.go.jp/kaihatsu/zenkokugakuryoku.html

National Institute for Educational Policy Research. (2014). Curriculum Research Center "National Assessment of Academic Ability" Assessment questions, correct answer examples, explanatory materials. https://www.nier.go.jp/kaihatsu/zenkokugakuryoku.html

National Institute for Educational Policy Research. (2015). Curriculum Research Center "National Assessment of Academic Ability" Assessment questions, correct answer examples, explanatory materials. https://www.nier.go.jp/kaihatsu/zenkokugakuryoku.html

National Institute for Educational Policy Research. (2016). Curriculum Research Center "National Assessment of Academic Ability" Assessment questions, correct answer examples, explanatory materials. https://www.nier.go.jp/kaihatsu/zenkokugakuryoku.html

National Institute for Educational Policy Research. (2017). Curriculum Research Center "National Assessment of Academic Ability" Assessment questions, correct answer examples, explanatory materials. https://www.nier.go.jp/kaihatsu/zenkokugakuryoku.html

National Institute for Educational Policy Research. (2018). Curriculum Research Center "National Assessment of Academic Ability" Assessment questions, correct answer examples, materials. https://www.nier.go.jp/kaihatsu/zenkokugakuryoku.html

Nisawa, Y. (2018a). Vorträge zur Mathematikdidaktik und zur Schnittstelle Mathematik/Mathematikdidaktik auf der gemeinsamen Jahrestagung GDM und DMV 2018 [Junior high school students' understanding of mathematical functions]. Jahrestagung der Gesellschaft für Didaktik der Mathematik, 52, 1315-1318.

Nisawa, Y. (2018b). Applying van Hiele's levels to basic research on the difficulty factors behind understanding functions. International Electronic Journal of Mathematics Education, 13(2), 61-65. https://doi.org/10.12973/iejme/2696

Nisawa, Y. (2018c). Basic research for teaching material development in function education-Focusing on function guidance system. Japan Journal of Mathematics Education and Related Fields, 59, 1-10.

OECD (Organization for Economic Cooperation and Development) (2017). PISA 2015 assessment and analytical framework: Science, reading, mathematic, financial literacy and collaborative problem solving (revised ed.). PISA, OECD Publishing. https://www.oecd.org/publications/pisa-2015-assessment-and-analytical-framework-9789264281820-en.htm

Okamori, K. (1983). Research and practice of mathematics education. Daiichihoki-syupan.

Shimizu, S. (Ed.) (2015). Fun with MATH, 4A 4B. Keirinkan.

Vidler, D. C. (1977). Achievement motivation. Academic Press.

Yokochi, K., \& Kikuchi, T. (Eds.) (1962). Modernization of mathematical functions in elementary and junior high schools. Meijitosho. 